\title{
Dimmer and neon transformer as a power controllable generator for atmospheric pressure plasma jet
}

\author{
Rosnah Mohd Zin¹, Chin Fhong Soon ${ }^{2}$, Nadhia Mohd Yusof ${ }^{3}$, Elfa Rizan Rizon ${ }^{4}$, Marlia Morsin ${ }^{5}$, \\ Kian Sek Tee ${ }^{6}$, Mohd Khairul Ahmad ${ }^{7}$, Nafarizal Nayan ${ }^{8}$ \\ 1,2,3,4,5,6,7,8 Faculty of Electrical and Electronic Engineering, Universiti Tun Hussein Onn Malaysia, Malaysia \\ 2,7,8 Biosensor and Bioengineering Laboratory, Universiti Tun Hussein Onn Malaysia, Malaysia
}

\begin{tabular}{l} 
Article Info \\
\hline Article history: \\
Received Nov 29, 2018 \\
Revised Jan 2, 2019 \\
Accepted Jan 18, 2019 \\
\hline
\end{tabular}

\section{Keywords:}

Atmospheric pressure plasma

jet

Dimmer

Neon transformer

Plasma torch

\begin{abstract}
The feasibility of a dimmer and neon transformer to provide power control to the atmospheric pressure plasma jet (APPJ) is presented in this paper. The standard neon transformer was previously shown applicable to power up a plasma torch to generate plasma irradiation of an APPJ at $8.64 \mathrm{kv}$ and $35 \mathrm{kHz}$. However, the plasma emission is not controllable. In this study, a lamp dimmer was proposed to function as a power controller to the neon transformer which drives the generation of plasma and discharged with argon gas. With the dimmer output voltage adjustable from $60-220 \mathrm{~V}$, the power and current of the dimmer were variable from 8.4 to $83.6 \mathrm{~W}$ and 0.08 and $0.38 \mathrm{~A}$, respectively. The temperature measured at the quartz tube and sample exposure site were less than $50^{\circ} \mathrm{C}$ suggested that the APPJ produced were non-thermal. A simple test by exposing plasma plume to a leaf sample showed that the plasma produced were non-damaging to the leaf. This portable system can provide control to the APPJ and applicable for bioengineering studies.
\end{abstract}

Copyright (c) 2019 Institute of Advanced Engineering and Science. All rights reserved.

\section{Corresponding Author:}

Chin Fhong Soon,

Faculty of Electrical and Electronic Engineering,

Universiti Tun Hussein Onn Malaysia,

86400 Parit Raja, Batu Pahat, Johor, Malaysia,

Email: soon@uthm.edu.my

\section{INTRODUCTION}

Plasma has wide application in plasma cleaning, surface modification, modifying adhesion properties and etching of semiconductor, packaging material processing, plasma medicine and food sterilization [1-2]. Plasma is a highly ionized and electrically conductive gas with positive ions, free electrons and radicals produced at low pressures or high temperature generally. Generally, plasma is a good electrical conductor and the density of the plasma can be controlled by the electric and magnetic fields [3]. The amount of plasma produced depends on the degree of ionization and temperatures. Different type of gases such as Helium, Argon, Nitrogen, Carbon dioxide and air can be used to produce plasma [2]. Plasma generation is divided into thermal and non-thermal (cold atmospheric plasma) type. Thermal plasma is induced by electrons and heavy particles at the same temperature under thermal equilibrium. Contrary, non-thermal plasmas have the ions and electrons at relatively lower temperature or down to room temperature [2].

In a vacuum chamber, low pressure plasma generates high concentration of reactive species that are functional to etch or deposit thin films. The disadvantages of the system are high maintenance cost and the size of the object to be plasma-treated is limited. To have an economic plasma system and wider application area, atmospheric-pressure plasma is an alternative that can be sought out. For generation of plasma at atmospheric pressure, high electrical potential is required. In the basic setup of a plasma torch of an APPJ system, a conductive electrode (anode) is inserted to a quartz tube and a ground (cathode) electrode is wrapped around the quartz tube. A ground plate can also be used to function as the cathode of the APPJ 
system. Usually, quartz is used as a dielectric material which limits the current that flows out of the tube. The pair of electrodes are used to energize and discharge the carrier gases and releasing plasma jet consisting of free electrons, ions, ultra-violet rays, ozone and reactive oxidative species [2]. The electric field controls the electromagnetic radiation of the plasma [3]. Sometimes, small percentage of oxygen is added to increase the generation of chemically reactive atoms and molecules.

There are several common sources of power supplies that could energize the electrodes of plasma torch at several kilovolts [2]-[5]. These plasma power supplies include RF pulsed high voltage amplifier [2], zero voltage switching driver/flyback transformer [4], DC pulsed power supply [5], autotransformer [6] and high voltage mixer amplifier [7]. They are usually heavy, bulky and costly. To energize a simple plasma system with a variable power supply, a high voltage power supply consists of a lamp dimmer driving a neon transformer is proposed. The objective of this paper was to investigate the feasibility of applying a lamp dimmer to control the power delivered to a plasma torch. With the variable AC power supply to the neon transformer, the emission of the electromagnetic radiations of the plasma jet can be controllable and studied for different applications. In this work, the effects of varying the input AC power supply of the lamp dimmer to the power supply to a neon transformer and temperature of the plasma exposure site will be investigated.

\section{RESEARCH METHOD}

A quartz tube with an inner diameter and outer diameter of 3 and $5 \mathrm{~mm}$, respectively was used as the body of the plasma torch (Figure 1(a)). A copper electrode was inserted to the gas inlet coupled to the top end of the quartz tube while the cathode is a thin copper tape wound around the quartz tube. As shown in Figure 1(b), a plasma torch was discharged with pure argon gas at a flow rate of $50 \mathrm{~L} / \mathrm{min}$. The high voltage generator consists of a $\mathrm{AC}$ dimmer connected to a $8.6 \mathrm{kp}-\mathrm{pV}$ neon transformer with an output power of $60 \mathrm{~W}$ and $36 \mathrm{kHz}$. The boosted voltage from the neon transformer was supplied to a plasma torch. According to its specification, the neon transformer accepts input ranging between $110 \mathrm{~V}$ and $240 \mathrm{~V}$ at 50/60 Hz. The dimmer supplies $\mathrm{AC}$ voltages varying from $60 \mathrm{~V}$ to $230 \mathrm{~V}$ by adjusting the potentiometer of the lamp dimmer. The distance between the discharged plasma plumes to the sample stage (a glass slide) is approximately $15 \mathrm{~mm}$ (Figure 1(b)). The whole system was setup with a variable power controlled by a dimmer to produce APPJ at room temperature.

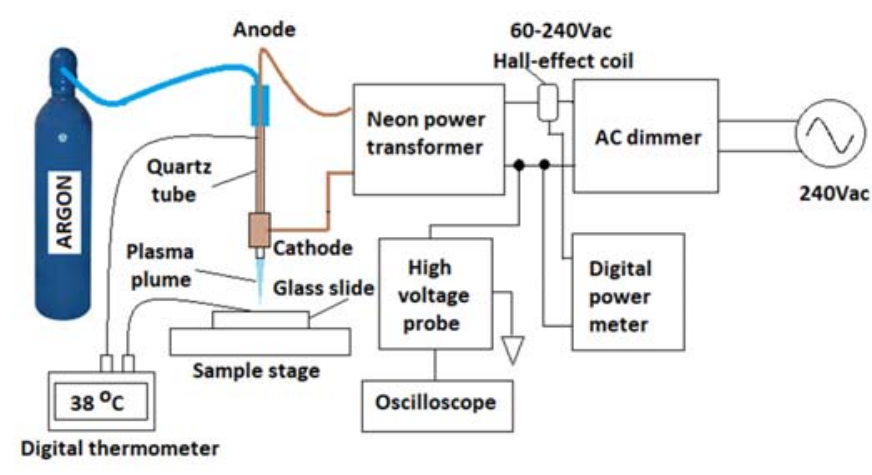

(a)

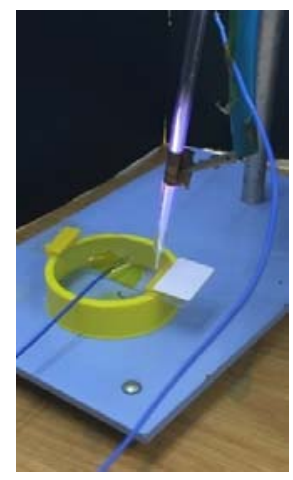

(b)

Figure 1. (a) The block diagram of the atmospheric pressure plasma jet system driven by a controllable power output from an AC dimmer and neon power transformer. (b) The plasma discharge produced.

For the electrical characterisation, a high voltage probe (P6015A, Tektronix, USA) of a digital oscilloscope (Agilent DSOX2022, USA) and a power meter (PZEM-061, ebay.com) were used to measure the current, voltage and power supplied to the neon sign transformer. The root means square (RMS) current output from the dimmer was measured using a hall-effect magnetic coil wrapped around the output wire of the dimmer to the neon transformer. The ground probe of the high voltage probe was connected to the ground terminal of the oscilloscope. A four-channel hand-held digital thermometer with K-Type thermocouple sensor was used to measure the temperature of the glass slide and quartz tube. The temperature was taken every 10 seconds with plasma irradiation for approximately 60 seconds. 


\section{RESULTS AND ANALYSIS}

The current of the lamp dimmer shows dimming curves in a sigmoid curve with respect to the adjusted voltage of the dimmer (Figure 2(a)). The power outputs of the lamp dimmer increased quadratically with respect to different control voltages. For the variable dimmer output voltage ranging from 60 to $220 \mathrm{~V}$, the power and current outputs of the dimmer were changed from 8.4 to $83.6 \mathrm{~W}$ and 0.08 and $0.38 \mathrm{~A}$, respectively. The current delivered to the neon transformer was relatively low. In the experiment, it was found that no plasma was dischargeable when the dimmer voltage was less than $60 \mathrm{~V}$ or at $10 \mathrm{~W}$. This is reasonable because the typical power requirement of the neon transformer is $60 \mathrm{~W}$. Although, the dimmer works on varying the effective Vp-p based on the phase angle of first and second half cycle of waveform, it can deliver variable Vrm output to the neon transformer (Figure 2(a)). Figure 2(b) shows the effects of varying the voltage output of the dimmer to the temperature of the plasma exposure site on the glass slide. As the voltage varied from 60 to $220 \mathrm{~V}$, the temperature of the plasma exposure site increased linearly from $35^{\circ}-53^{\circ} \mathrm{C}$. This is approximation to the characteristic of non-thermal plasma [2] and induced non-damaging effects to the sample under irradiation of the APPJ. The detrimental effect of the plasma output maybe related to the power and current deliver to the plasma torch. According to a previous study [8], the current delivered from the neon transformer to the plasma torch at approximately $9.8 \mathrm{mAp}$-p was shown to be non-destructive to the sample. To produce plasma with destructive effect, higher current of a few amperes should be applied to the plasma torch [4].

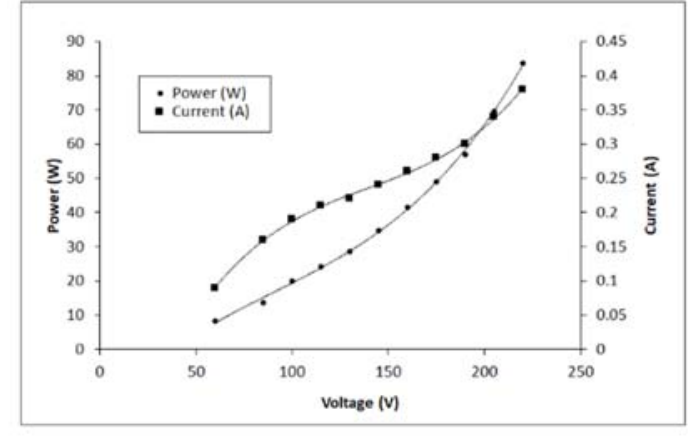

(a)

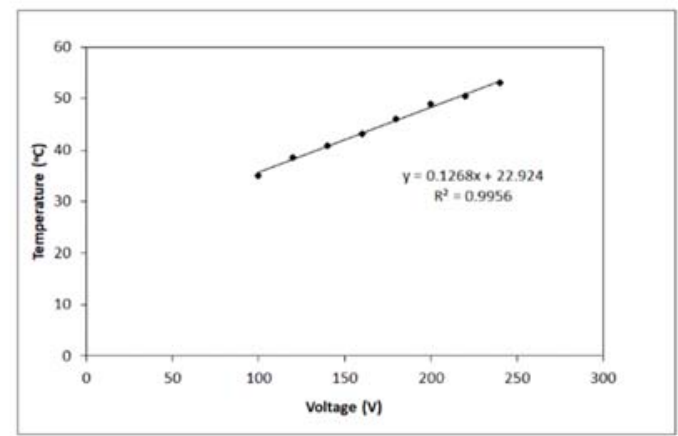

(b)

Figure 2. (a) The relationship of power and current to the output voltage of the dimmer, (b) Temperature measured adjacent to the plasma exposure site on the glass slide

The temperature of the quartz tube and glass surface were monitored over time upon the onset of the plasma torch and argon gas infusion. A comparison was made between the temperature measured for quartz tube and glass slide. Interestingly, the temperatures determined for both surfaces indicated with a cross-over at 20 seconds (Figure 3). During the first 20 seconds when the plasma torch was energized at $6 \mathrm{kV}$ and $33 \mathrm{kHz}$, the quartz temperature was found higher than the glass surface temperature which is related to the accumulation of electrons and ionized gas at the anode of the plasma torch. This caused a higher temperature of $50^{\circ} \mathrm{C}$ measured at the quart tube of the plasma torch. The temperature of the glass slide was lower at $35^{\circ} \mathrm{C}$. The emission of the plasma plume was observed with instable discharges for an operation time less than 20 seconds. The full discharge of the electrons and ionized gas reached the surface of the glass slide caused a raise of temperature from 35 to $55^{\circ} \mathrm{C}$ after 20 seconds of operation time. The temperature at the quartz tube surface decreased slightly to $42^{\circ} \mathrm{C}$. The result presented in Figure 3 indicates that the plasma torch needs to be activated at least 20 seconds for stabilization before application to samples. 


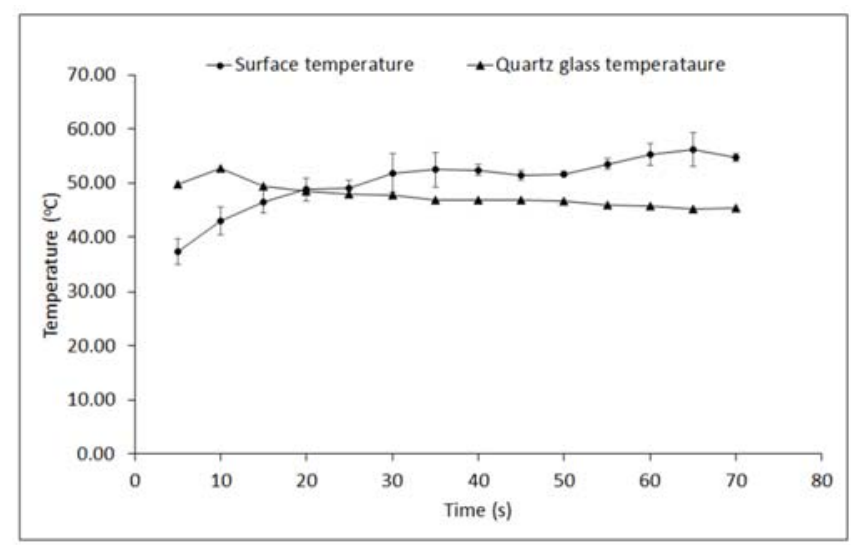

Figure 3. Temperature measured at the surfaces of glass slide and quartz tube at input voltage $240 \mathrm{Vac}$

Figure 4 shows the typical voltage waveform outputs of a lamp dimmer when the plasma torch was switched off and without purging the argon gas. The waveforms were swinging in between $340 \mathrm{Vp}-\mathrm{p}$. The dimmer could turn on the output at an adjustable time or changing the phase angle of each alternating half cycle of the sinusoidal waveforms and thus, varying the effective root means square value and power output. At low voltage of $60 \mathrm{~V}$, a major cycle of the full sinusoidal waveforms was "chopped" while maintaining the peak voltage of the waveform as shown in Figure 4(a). Figure 4(a) shows that the output waveform was turned on after $0.01 \mathrm{sec}$ when the sinusoidal crosses zero voltage. The later the dimmer is fired or starts to conduct, thus, less power is supplied to the plasma torch. As the controlled voltage increased from $60-220 \mathrm{~V}$, the sinusoidal waveforms were gradually recovered. The modulation of the waveforms is related to the dimmer control circuit. The dimmer circuit consists of a diac, variable resistor and capacitor that control the switch-on of the triac once reaching the peak of the input sinusoidal waveform. For a lamp dimmer circuit, the waveforms produced are partial cycle of the sinusoidal waveforms with leading edges. The dimmer switches on and off based on phase angle and therefore, less power is lost.

The results presented in Figure 4 were recorded without switching on the plasma torch and neon transformer. Once the inductive load or transformer was switched on at voltages less than $120 \mathrm{~V}$, overshoot voltage spikes could be observed due to the capacitive nature of the neon transformer as shown in Figure 5ac. The interference affects the physical irradiation of the plasma plume that was observed to be unstable once the dimmer voltage was reduced below $120 \mathrm{~V}$. The phase angle waveforms of the dimmer with the neon transformer load (Figure 5(d-i)) were similar to the waveforms of the dimmer without the plasma torch load (Figure 4(d-i)). This indicates that the dimmer works for inductive load when the dimmer voltage is more than $120 \mathrm{~V}$. Figure 6 shows a typical voltage waveform of the neon transformer measured at the cathode of the plasma torch. The envelops of waveform (Figure 6) seemed to be similar to amplitude modulated waveforms in which, it was formed by multiple sinusoidal waveforms. The composite waveforms cycle repetively when the dimmer supply voltage is $>150 \mathrm{~V}$. 

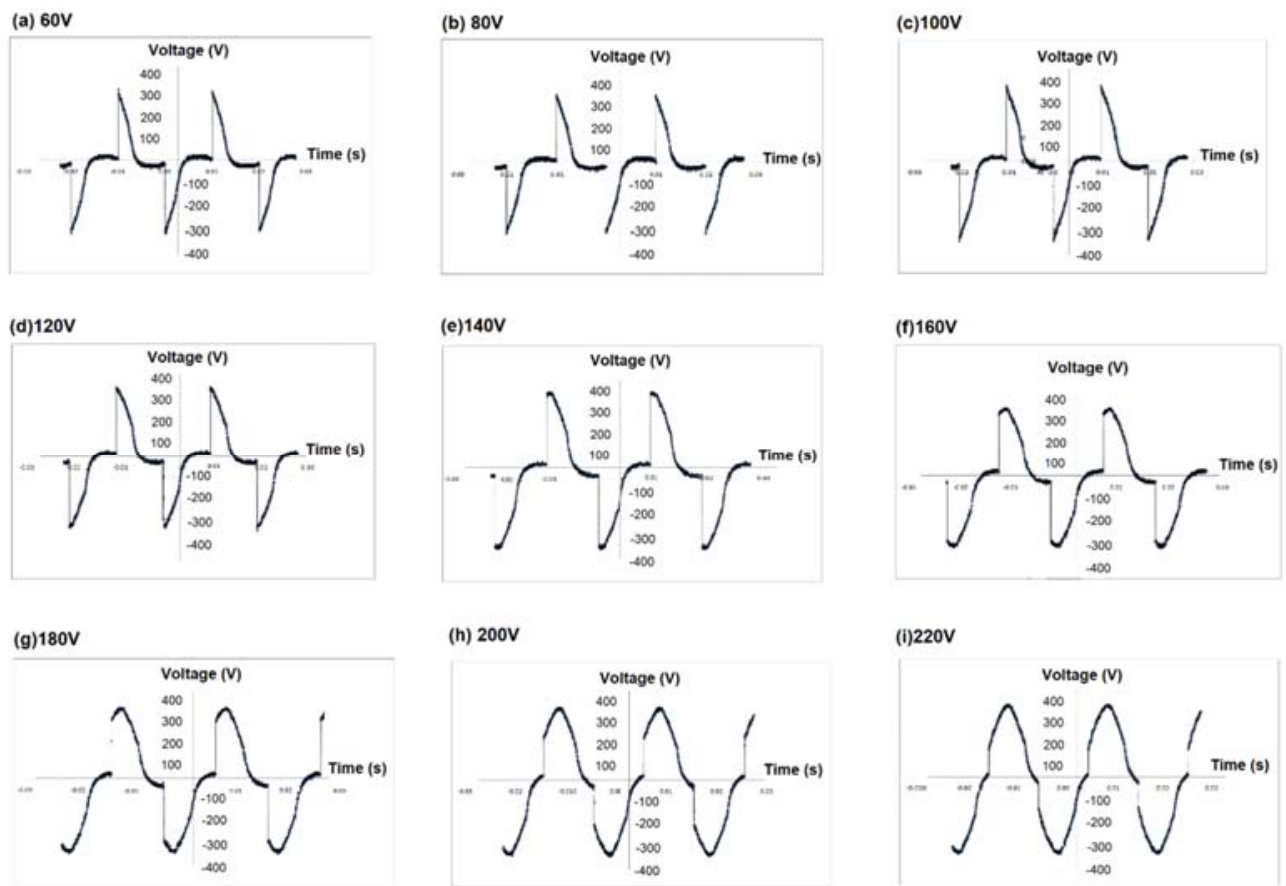

Figure 4. The output waveforms of the dimmer transformer by varying dimmer voltages at (a) $60 \mathrm{~V}$, (b) $80 \mathrm{~V}$, (c) $100 \mathrm{~V}$, (d) $120 \mathrm{~V}$, (e) $140 \mathrm{~V}$, (f) $160 \mathrm{~V}$, (g) $180 \mathrm{~V}$, (h) $200 \mathrm{~V}$, (i) $220 \mathrm{~V}$.
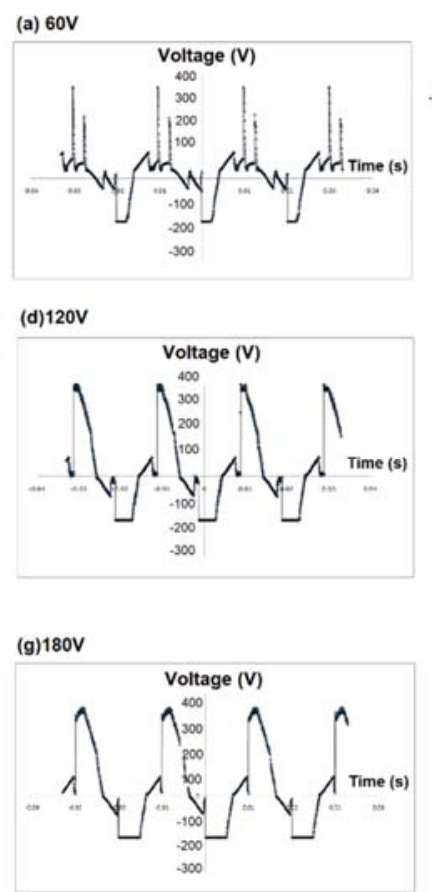

(b) $80 \mathrm{~V}$

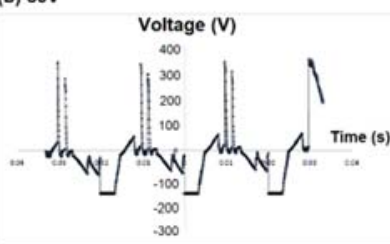

(e) $140 \mathrm{~V}$

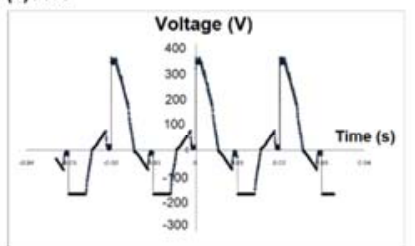

(h) $200 \mathrm{~V}$

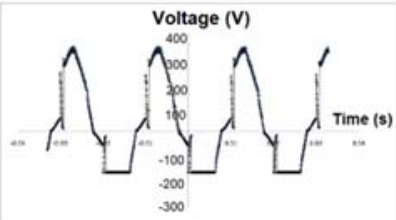

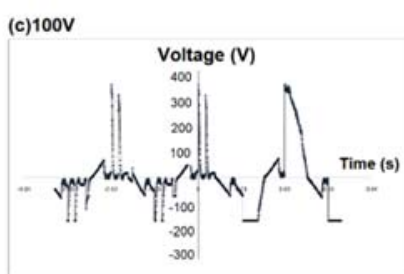

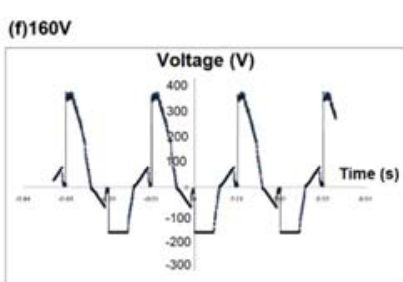

(i) $220 \mathrm{~V}$

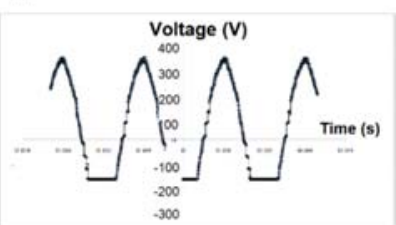

Figure 5. The output waveforms of the neon transformer by varying dimmer voltages at (a) $60 \mathrm{~V}$, (b) $80 \mathrm{~V}$, (c) $100 \mathrm{~V}$, (d) $120 \mathrm{~V}$, (e)140V, (f) $160 \mathrm{~V}$, (g) $180 \mathrm{~V}$, (h) $200 \mathrm{~V}$, (i) $220 \mathrm{~V}$. 
To verify if plasma emitted is destructive, the output of the plasma was irradiated to a leaf for 30 seconds at full power (dimmer input at 220V). Plausibly, the leaf was not found with any burn except a little dryness due to the gas flow (Figure 7). This is suggesting that the plasma plume produced by the proposed system was not causing damaging effects to a living organism. A previous study shown that the APPJ can set to modify the surface properties or wettability of fabric without damaging effects [8]. The result shows that neon transformer-based plasma power generator has the advantage of delivering plasma jet at low current and heat to the sample especially cells [9-10]. Such a non-thermal APPJ has great potential for biotechnology or textile industry.

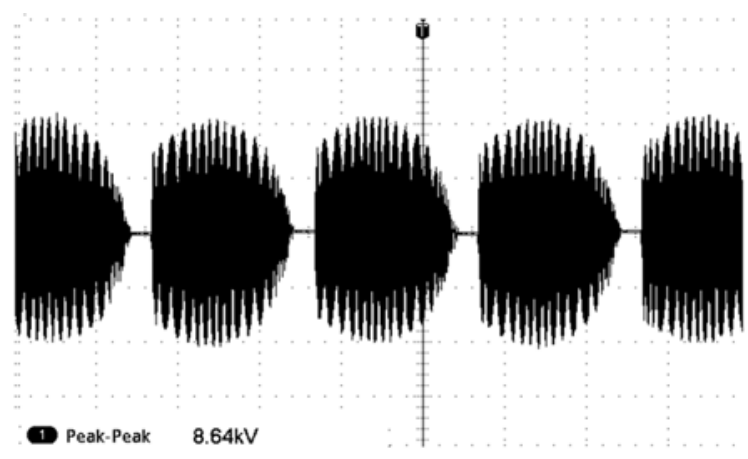

Figure 6. The output waveforms of the neon transformer with a Vp-p of $8.64 \mathrm{kV}$

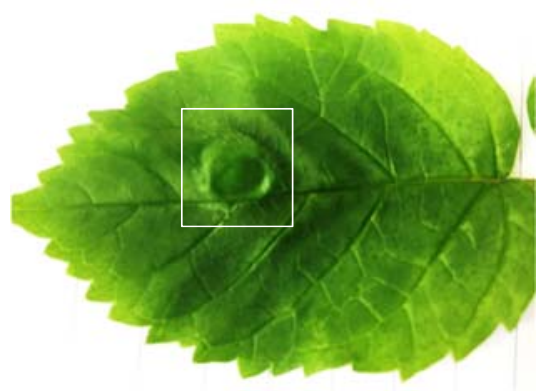

Figure 7. A leaf after exposure to plasma plume of an APPJ for 30 seconds. The white box marked the plasma exposure area of the leaf.

\section{CONCLUSION}

The dimmer circuit applied for lamp brightness control is suitable to be used to control the plasma emission of an APPJ ranging from input voltage of $60-220 \mathrm{~V}$. The power output of the dimmer was variable between 8.4 to $83.6 \mathrm{~W}$ when the input voltage of dimmer was varied between $60-220 \mathrm{~V}$. This was achieved by controlling the phase angle of the sinusoidal output of the dimmer. This range of power was sufficient to regulate the output power of the neon transformer which requires a nominal power of $60 \mathrm{~W}$. However, the discharge of plasma caused interference to waveforms of the dimmer output for dimmer input voltage less than $100 \mathrm{~V}$ and lead to instable plasma discharge. The temperature of the irradiated site or glass slide is linearly proportional to the control voltage of the dimmer. Based on the temperature at the exposure site ranging from $35-53^{\circ} \mathrm{C}$ and low current application to the plasma torch $(\sim 9.8 \mathrm{mApp})$, the plasma produced with the proposed plasma system is non-damaging to the sample irradiated. The electrical properties characterized for the improved APPJ driven by dimmer and neon transformer presented potential to be applied in biotechnology.

\section{ACKNOWLEDGEMENTS}

This project was supported by Short Term Grant (STG) Vot No. U641 awarded by Universiti Tun Hussein Onn Malaysia.

Dimmer and neon transformer as a power controllable generator for ... (Rosnah Mohd Zin) 


\section{REFERENCES}

[1] Dayun Yan, et al., "Cold atmospheric plasma, a novel promising anti-cancer treatment modality," Oncotarget, Vol. 8(9), pp.15977-15995, 2017.

[2] Vladimir Scholtz, et al., "Nonthermal plasma-a tool for decontamination and disinfection," Biotechnology Advances, Vol. 33(6), pp. 1108-1119, 2015.

[3] S. Zerouali, et al., "Non-linear control techniques applied to high frequency inverters for induction plasma generator," International Journal of Advanced Science and Technology, Vol. 69, pp. 33-46, 2014.

[4] Rosnah Mohd Zin, et al., "Zero voltage switching driver and flyback transformer for generation of atmospheric pressure plasma jet", AIP Conference Proceedings, Vol. 1883(1), pp. 020023, 2017.

[5] Denis P. Dowling, et al., "Influence of DC pulsed atmospheric pressure plasma jet processing conditions on polymer," Activation Plasma Process Polymer, Vol. 8, pp. 718-727, 2011.

[6] L Giuliani, et al., "Electrical characterization of an air microplasma jet operated at a low frequency ac voltage," Journal of Physics. Conference Series, Vol. 370, pp. 012011, 2012,.

[7] Rizan Rizon Elfa, et al., "Comparative study between chemical and atmospheric pressure plasma jet cleaning on glass substrate," AIP Conference Proceedings, Vol. 1788, pp. 030113, 2017.

[8] Rizon Elfa, et al., "Atmospheric pressure plasma jet treatment of malaysian batik fabrics," Rizan Journal of Telecommunication, Electronic and Computer Engineering, Vol. 9(3), pp. 89-92, 2017.

[9] Rizan Rizon Elfa, et al., "Electrical and optical characteristic of atmospheric pressure plasma needle jet driven by neon transformer," AIP Conference Proceedings, Vol. 1883(1), pp. 020026-1-020026-6, 2017.

[10] Hongbin Liu, et al., "A novel DC-driven atmospherical-pressure cold microplasma source for biomedical application," IEEE Transaction on Radation and Plasma Medical Sciences, Vol. 1(5), 2017.

\section{BIOGRAPHIES OF AUTHORS}
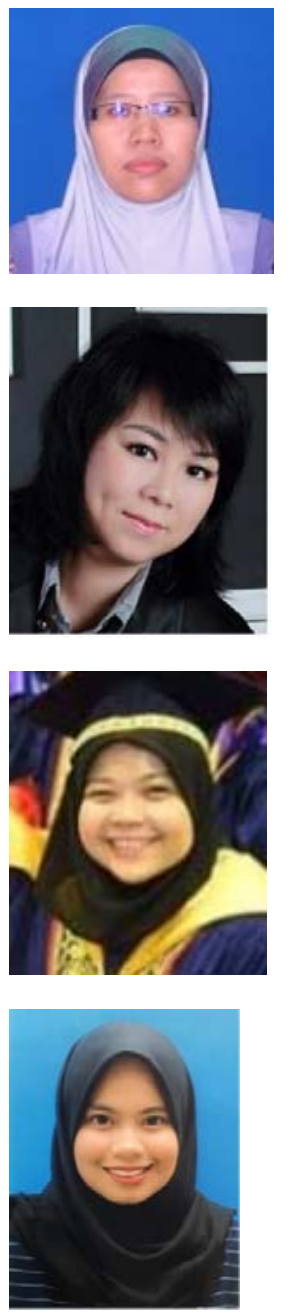

Rosnah Mat Zin is a senior lecturer at the Faculty of Electrical and Electronics Engineering, Universiti Tun Hussein Onn Malaysia. She is graduated with a master's degree in electrical engineering from Universiti Tun Hussein Onn Malaysia. She has many years of teaching experience in electronics courses.

Dr. Soon Chin Fhong is an associate professor at the Faculty of Electrical and Electronics Engineering, Universiti Tun Hussein Onn Malaysia. She holds a PhD in Biomedical Engineering from University of Bradford, United Kingdom. Her research interest is in application of plasma physics in bioengineering, 3D bioprinter, wireless applications, microencapsulation and microfluidic development. She is currently a principle researcher at Microelectronic and Nanotechnology Shamsuddin Research Center (MiNT-SRC).

Elfa Rizan Rizon was a research student at the Faculty of Electrical and Electronics Engineering, Universiti Tun Hussein Onn Malaysi and she holds a master's degree in electrical engineering. She is currently working at Intel Manufacturinig Sdn. Bhd., Bayan Lepas, Penang.

Nadhia Mohd Yusof is a research research assistant with Microelectronic and NanotechnologyShamsuddin Research Center. She is graduated with a bachelor's degree in electrical engineering from Universiti Tun Hussein Onn Malaysia. 


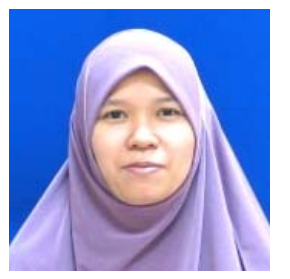

Dr. Marlia Morsin is currently a senior lecturer at Universiti Tun Hussein Onn Malaysia. She holds a PhD in Micro and Nanoelectronics from Universiti Kebangsaan Malaysia. Her research interest is in the application of gold nanoparticles and the simulation of surface plasmon resonance in sensor development.

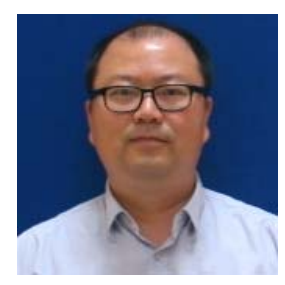

Ir. Dr. Tee Kian Sek is a senior lecturer at Universiti Tun Hussein Onn Malaysia. He holds a $\mathrm{PhD}$ in Mechatronics Engineering from Leeds University, United Kingdom. He is professional engineer registered with the Board of Engineers Malaysia (BEM) has 8 years of working experience with the industry. His rearach interest is in power electronics, robotics, ergonomics and human gait analysis.

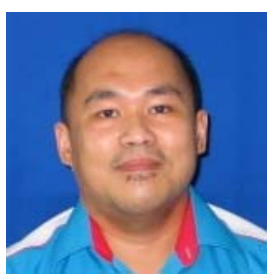

Dr. Mohd Khairul Ahmad is an associate professor at the Faculty of Electrical and Electronics Engineering, Universiti Tun Hussein Onn Malaysia. He holds a $\mathrm{PhD}$ in Electronics from Shizuoka University, Japan. His research interest is in Dye-sensitised solar cells and synthesis of nanostructured materials. He is a principle researcher at Microelectronic and Nanotechnology Shamsuddin Research Center (MiNT-SRC).

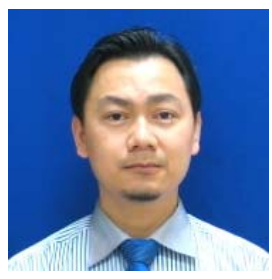

Dr Nafarizal Nayan is an associate professor at the Faculty of Electrical and Electronics Engineering, Universiti Tun Hussein Onn Malaysia. He holds a $\mathrm{PhD}$ in Electrical Engineering from Nagoya University in Japan and he is currently the Head of Microelectronic and Nanotechnology Shamsuddin Research Center (MiNT-SRC). His research interest is in new plasma processing system, application of plasma in biomedical engineering, diagnostic of plasma properties, nanoscale analysis and imaging. 